\title{
Future of muscle research in diabetes: a look into the crystal ball
}

\author{
Michael Roden ${ }^{1,2,3}$
}

Received: 2 March 2015 / Accepted: 7 April 2015 / Published online: 24 May 2015

(C) Springer-Verlag Berlin Heidelberg 2015

\begin{abstract}
In type 2 diabetes, skeletal muscle is not only responsible for early metabolic abnormalities, but its contractile activity also offers an efficient prevention and treatment strategy. This outlook into the coming decades summarises challenges and opportunities for translational research on skeletal muscle in diabetes and related diseases. Currently, our understanding of the interactions between myocellular networks, the master regulators of resting metabolism, and muscle's position within multi-organ crosstalk, is incomplete. In the face of an ageing population, changes within muscle tissue appear to be the predominant mechanisms responsible for sarcopenia, but the relative roles of obesity and ageing as driving forces of its development are less clear. To address these research questions, innovative approaches to optimising exercise training or minimising sedentarism will need to be devised and tested in large-scale standardised prospective studies. Finally, another major challenge will be the identification and evaluation of muscle targets to prevent and treat metabolic diseases. This is one of a series of commentaries under the banner ' 50 years forward', giving personal opinions on future perspectives in diabetes, to celebrate the 50th anniversary of Diabetologia (1965-2015).
\end{abstract}

Michael Roden

michael.roden@ddz.uni-duesseldorf.de

1 Department of Endocrinology and Diabetology, Medical Faculty, Heinrich Heine University Düsseldorf, c/o Auf'm Hennekamp 65, D-40225 Düsseldorf, Germany

2 Institute for Clinical Diabetology, German Diabetes Center, Leibniz Institute for Diabetes Research at Heinrich Heine University, Düsseldorf, Germany

3 German Center for Diabetes Research, Partner Düsseldorf, Düsseldorf, Germany
Keywords Exercise mimetics · Insulin resistance · Lipotoxicity $\cdot$ Mitochondria $\cdot$ Multiomics $\cdot$ Myogenesis · Myokines $\cdot$ Sarcopenia $\cdot$ Stem cells

$\begin{array}{ll}\text { Abbreviations } \\ \text { AMPK } & \text { AMP kinase } \\ \text { DAG } & \text { Diacylglycerol } \\ \text { ER } & \text { Endoplasmic reticulum } \\ \text { IKK } & \text { IkB kinase } \\ \text { miRNA } & \text { MicroRNA } \\ \text { MR } & \text { Magnetic resonance } \\ \text { mTOR } & \text { Mammalian target of rapamycin } \\ \text { NFKB } & \text { Nuclear factor } \text { B B } \\ \text { PGC1 } & \text { PPAR } \gamma \text { coactivator } 1 \\ \text { PPAR } & \text { Peroxisome proliferator-activated receptor } \\ \text { PPR } & \text { Pattern recognition receptor }\end{array}$

\section{Background}

Almost 50 years ago, Sir Philip Randle suggested that increased lipid mobilisation from adipose tissue provides NEFA for oxidation in skeletal muscle, which would compete with glucose oxidation [1]. While studies in humans have since shown that this 'glucose fatty acid cycle' fails to explain common insulin resistance [2,3], his ground-breaking experiments paved the way for research on muscle metabolism.

\section{Why should muscles be relevant for diabetes?}

Skeletal muscle contributes both to the storage of ingested energy and the depletion of energy stores. Muscles account for more than two-thirds of excess glucose disposal after meal 
ingestion and nearly all non-oxidative glucose storage as glycogen during hyperinsulinaemia [3]. As an ectopic lipid compartment, muscle also stores NEFA derived from excess fat intake or augmented adipocyte lipolysis [2, 3]. Finally, muscle contraction rapidly burns excess stored energy and improves insulin resistance. Although novel roles are being elucidated for adipose tissue dynamics and the intestinal microbiota, muscle remains the main organ directly responsible for the earliest metabolic abnormalities leading to type 2 diabetes.

Rapid developments in molecular biology and refined clinical methodology have triggered major breakthroughs in muscle research. Nevertheless, a full understanding of cellular and inter-organ networks and their relevance for healthy ageing and diabetes treatment remains elusive. Unsurprisingly, clinical guidelines are incomplete, and numerous issues still need to be addressed; this offers considerable opportunities for future muscle research.

\section{Better understanding muscle metabolism and insulin action}

Comprehensive metabolic testing, including magnetic resonance (MR) spectroscopy, has identified a reduction in insulin-dependent muscle glucose transport as the primary abnormality underlying insulin resistance $[2,3]$. Nevertheless, there has been debate over the extent to which muscle contributes to hyperglycaemia and could therefore be the main culprit causing type 2 diabetes. Knockout mice and genome-wide association studies have failed to establish tight links between muscle insulin resistance and overt diabetes. Recently, however, a Greenlandic study demonstrated that a gene variant encoding TBC1D4 (AS160) impairs insulinstimulated muscle GLUT4 mobilisation, resulting in postglucose hyperglycaemia and tenfold higher type 2 diabetes risk [4]. Small-scale studies have shown that inherited mitochondrial abnormalities may also contribute to insulin resistance (for example, [5]), but confirming the role of muscle as a culprit of type 2 diabetes will require prospective studies in carefully selected cohorts undergoing comprehensive geno-/ phenotyping.

More is known on the role of muscle as a 'victim' during the development of insulin resistance. Decades of experiments simulating energy excess have led to few mechanistic concepts: toxic metabolites, inflammation, mitochondrial abnormalities and endoplasmic reticulum (ER) stress (Fig. 1). The paradigm of 'lipotoxicity' postulates that elevated lipid intermediates in muscle interfere with insulin signalling. In humans, the sequence of events is best documented for a specific $\mathrm{C} 18$-diacylglycerol (DAG) that activates protein kinase C- $\theta$ (PKC- $\theta$ ), thereby inducing inhibitory serine-1101 phosphorylation of IRS-1 [6]. However, saturated NEFA can also cause insulin resistance by binding to toll-like receptor 4
(TLR4) and activating IkB kinase (IKK)/nuclear factor $\mathrm{\kappa B}$ (NFKB) and the sphingolipid/ceramide pathway. Alternatively, excessive NEFA and amino acid fluxes exceeding muscle oxidative capacity may give rise to acylcarnitines and stimulate IKK/NFKB [3]. These pathways may further cause ER and oxidative stress, although the existence of a master regulator or a hierarchy in the sequence of events remains uncertain.

In the future, rapid advances in high-resolution imaging are likely to generate new tools for tracing cell function and molecular cascades. Imaging combined with specific labelling techniques will allow real-time monitoring of organelle dynamics. Large-scale use of fast-replicating or short-lived metazoa (worms or zebrafish) will be helpful for time-lapse studies in intact organisms. For human studies, new hardware and software solutions will optimise metabolic imaging, for example by combining multinuclei MR imaging/spectroscopy for real-time in vivo monitoring of metabolic fluxes and bioenergetics. This could lead to the realisation of the dream of a portable high-resolution molecular imaging device for clinical diagnostics.

These technologies should allow us to decode intracellular networks and to spot master regulators of muscle metabolism. In particular, the regulation of lipid droplet assembly and partitioning (aside from already known regulators such as adipose triglyceride lipase and perilipins) is likely to gain more attention [3]. This could allow us to design liposomes in vitro and subsequently modify these organelles in vivo to counteract insulin resistance by trapping lipotoxins. Refined assessment of the subcellular distribution of signal molecules (e.g. DAG or ceramides) could also ultimately explain the athletes' paradox of excessive triacylglycerol storage [2, 3]. In addition, we can expect the study of mitochondria to remain of interest, although clear test protocols for mitochondrial features will need to be established to satisfactorily define abnormalities in mitochondrial capacity and dynamics [2].

The positioning of muscle within organ crosstalk also needs to be revisited. Originally, muscle was thought to passively receive information from cytokines or metabolites originating from other organs. Although muscle has now been established to be a secretory organ releasing myokines (e.g. interleukins and fibroblast growth factor-21 [FGF21]) [7] and microRNAs (miRNAs) [7], the function and relevance of the complete muscle secretome remains to be discovered. Recent data suggest that differential expression and release of certain miRNAs play a role in insulin resistance and regulation by intrauterine undernutrition [8]. In addition, innate pattern recognition receptors (PPRs), which sense danger (nutrient)- and pathogen (intestinal microbiota)-associated molecular patterns, may serve as the link between microbiome diversity, lipotoxins, the inflammasome and insulin resistance [9]. Analysis of such high-level crosstalk will require the development of micromethods for the application of multiple tests in 


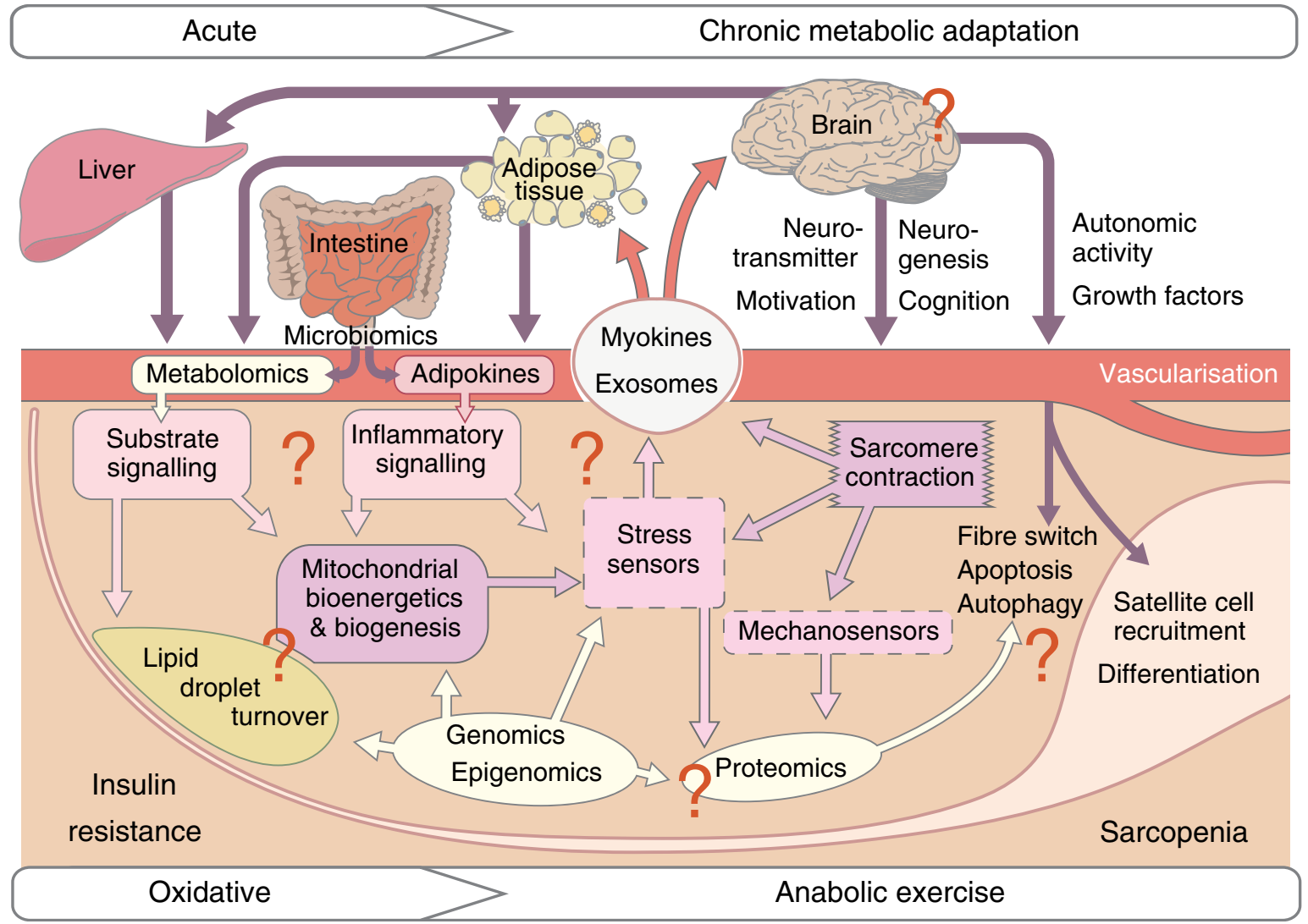

Fig. 1 The muscle in the centre of diabetes research. Question marks indicate present challenges and future opportunities for the better understanding of the (patho)physiology and treatment of muscle-related alterations in elderly, obese and diabetic patients

large-scale cohorts. The path could lead from identifying molecular signatures and 'multiomics' to commercially available 'diagnostic muscle panels'. Currently, several conceptual and methodological issues limit the predictive power of networkbased approaches [10]. However, diseases with overlapping network modules, such as obesity-related sarcopenia and type 2 diabetes, can be expected to benefit from 'interactome'based platforms, predicting common molecular pathways despite incomplete multiomics data.

\section{Focusing on muscle mass and sarcopenia}

The key drivers of type 2 diabetes, obesity and ageing, also associate with loss of muscle mass and functionality, termed sarcopenia. Unfortunately, current definitions of sarcopenic obesity are so heterogeneous that estimates of its prevalence range from $4 \%$ to $94 \%$ [11]. Differences in the pathogenesis of both ageing- and disease-related sarcopenia is insufficiently described and are unlikely to relate exclusively to either abnormal protein turnover or impaired oxidative capacity (Fig. 1). In diabetes, insulin resistance and myostatin/activin A activation probably contribute to muscle wasting; in addition, anabolic effects of androgens and growth hormone/IGF1 have been intensively studied $[11,12]$. Nevertheless, the evidence for the relative roles of anabolic (insulin signalling via mammalian target of rapamycin [mTOR]) and catabolic pathways (activation of ubiquitin/proteasome and autophagy/ lysosome via forkhead box $\mathrm{O}$ [FOXO] or NFkB) is scarce in human diabetes and obesity [12].

Understanding these mechanisms may help us discover methods for stimulating myogenesis and thus overcoming sarcopenia. A recent preliminary success in producing bioengineered human myobundles with three-dimensional structure and effective contractility may be an important step towards translation into improved muscle mass and function in patients [13]. However, in order to generate fully functioning human myobundles we will need to resolve the problems of delivering sufficient oxygen by capillary networks, regulating fibre-type composition and providing adequate electrical and mechanical activation.

\section{Testing contractile function and physical activity}

Despite widely published evidence of risk reduction and partial remission of type 2 diabetes by lifestyle modification, guidelines conclude that more studies are needed to determine whether total caloric expenditure, or exercise mode, intensity, duration or frequency, are the determining benefits of physical 
training [14]. As regards clinical diabetes treatment, insufficient knowledge on the interplay between various cellular and systemic effects (e.g. myokine release, increased perfusion or cardiopulmonary activation) limits the ability to predict glycaemic responses to acute exercise and risk of hypoglycaemia.

A unique feature of acute muscle contraction resides in the insulin-independent stimulation of glucose transport and ATP synthase flux, mediated mainly by AMP kinase (AMPK), calcium signalling and p38 MAP kinase (MAPK)/extracellular signal-related kinase (ERK) [15]. Much less is known about the mechanisms underlying the transition from acute to chronic effects and adaptation to repeated exercise during endurance (aerobic) or resistance (strength) training (Fig. 1). Endurance training seems to act primarily by reducing insulin resistance and improving mitochondrial biogenesis (via different pathways including AMPK, mitochondrial transcription factor A [TFAM], nuclear respiratory factor-1 [NRF1] and peroxisome proliferator-activated receptor $[\mathrm{PPAR}] \gamma$ coactivator $1[\mathrm{PGC} 1] \alpha$ ), while resistance training could increase protein synthesis via mTOR and eukaryotic initiation factor 4Ebinding protein 1 (EIF4EBP1) [15]. Fibre-type reprogramming, endocrine regulation and muscle-derived myokines add further dimensions to muscular adaptation. As with insulin resistance, redundancy of pathways leading to identical exercise responses makes it difficult to single out central regulators.

Despite its obvious beneficial health effects, exercise training results in surprisingly modest improvements in glycaemic control (a reduction in $\mathrm{HbA}_{1 \mathrm{c}}$ of $0.5-0.7 \%$ ) in type 2 diabetes [14]. Moreover, certain training programmes may independently improve either metabolic or bioenergetic function. Recent findings point to an inherited predisposition defining individual success in exercising: in first-degree relatives of type 2 diabetes patients, one individual single nucleotide polymorphism (SNP) in the gene encoding NADH dehydrogenase (ubiquinone) 1 beta subcomplex 6 (NDUB6), a subunit of mitochondrial complex 1, modified their response of muscle mitochondrial function to acute and chronic exercise [16]. Despite evidence for the impact of other gene variants (e.g. PPAR $\delta / \gamma, \operatorname{PGC} 1 \alpha$ ), a direct causal relationship between the function of these genes and muscle metabolism is still uncertain. Other geneenvironment interactions, such as epigenetic modulation (DNA methylation or histone acetylation) and miRNA expression and uptake/release by exosomes, are already evolving as hot topics that may enable us to better understand the individual variability of training responses. The observation that type and duration of exercise differentially regulate circulating miRNA levels could qualify them as biomarkers of training efficacy or muscle fitness, but we would first need better standardisation of blood sampling and measurement.
Improving the efficacy of exercise training in persons at risk of diabetes requires innovative concepts. Molecular research will be necessary in order to gain mechanistic insight into metabolic switches at the start and end of exercising and at the transition from acute to chronic exercising. Of note, normal responses and adaptations to exercising have been observed even during the experimental elimination of one or more cellular pathways. This could point to the maintenance of homeostasis as being a central topic for future exercise physiology research [15]. For type 2 diabetes, new training modes should be designed by addressing the timing of exercise (chronoactivity), variable intensity, and hypoxic and eccentric protocols. Even shortening the periods of physical inactivity/sedentarism seems to improve body fat content and metabolism in newly diagnosed type 2 diabetes [17]. As alterations during physical inactivity are not necessarily the reverse of exercise effects, future studies should prospectively investigate whether simply reducing sedentarism has longterm benefits in obesity and diabetes. These investigations should not be limited to muscle but should also include assessing whole body functions, as well as satisfaction with and adherence to training. Large-scale controlled multicentre trials employing identical protocols are still desperately needed to increase the precision of guidelines on exercise in metabolic diseases. Finally, a multidisciplinary approach will be necessary to overcome the limited efficacy of exercise and 'resistance' to it, and to establish tailored lifestyle modification with the vision of personalised medicine. One can expect that the sports industry will support this by developing intelligent training devices, which could sense changes in physical condition and adapt muscle-group-specific resistance, aiming at optimised time-efficient training bouts. The growing elderly population will need tailored training tools, possibly including electrical stimulation, taking into account limited mobility.

\section{Identifying and evaluating muscle targets to treat metabolic diseases}

The present treatment of obesity and type 2 diabetes only indirectly addresses muscle insulin resistance by improving hyperglycaemia, dyslipidaemia or unfavourable cytokine profiles. Despite several pharmacological attempts, no drug directly targeting muscle has yet entered the market successfully. The above considerations result in a short list of therapeutic concepts, which are already in development or might be promising targets.

Modulators of lipid storage and signalling Small molecules reducing intracellular levels of lipotoxins by inhibiting, for example, DAG transferase or ceramide synthase, show some promising results in animal models. Modulation of lipid droplet formation aimed at reducing intracellular lipotoxins could 
be another strategy. Such strategies will need to take into account the risk of unwanted effects in tissues that depend on intact lipid metabolism, such as brain, adipose tissue and steroid-hormone-producing endocrine organs.

'Mitochondrial medicine' and exercise mimetics This concept aims at mimicking the effects of caloric restriction or exercise in mitochondria. Activators of AMPK, sirtuin-1 (e.g. resveratrol), PPAR $\delta / P G C 1 \alpha$ and/or nuclear receptors are already in (pre)clinical testing, while small molecules targeting mitochondrial bioenergetics and biogenesis are future hopes [18]. The big question is: will we be able to directly activate muscle mitochondrial function or mass in a controlled fashion, and what is the consequence of continuous stimulation of these pathways? Indeed, chronic activation of AMPK could inhibit protein synthesis and stimulate autophagy, and stimulating muscle PGC- $1 \alpha$ has been shown to cause muscle atrophy in mice [18]. Mitochondrial medicine also faces the challenge of drug effects resulting from the generally narrow therapeutic range, such as hyperthermia and multi-organ failure.

'Anti-sarcopenic' concepts Inhibition of myostatin/activin has been shown to be beneficial for muscle wasting and insulin resistance in several diseases including obesity and diabetes [12]. Pharmacological attempts to increase muscle mass, by modification of targets of mechanosensation, protein anabolism (e.g. mTOR), contractile function or mechanical stress (e.g. cAMP-response element binding protein [CREBP]) and calcium fluxes, warrant further evaluation. However, the therapeutic potential requires more than a demonstration of increased muscle mass in humans. Therapeutic trials will need to monitor muscle functionality and nitrogen balance as well as safety, particularly among the obvious target group, older immobilised patients, and the possible misuse by bodybuilders. Other possibilities, including preventing diabetesrelated damage, or stimulating or even transplanting muscle progenitor (satellite) cells within muscles, will need to meet high expectations. This is likely to involve exploiting the therapeutic potential of organ crosstalk by modulators of cytokines, PPRs and miRNAs or other yet unknown circulating factors identified through multiomics.

\section{No end in sight!}

Fifty years after Sir Philip Randle's publication, our understanding of lipid-glucose interaction has shifted towards a multidimensional network of pathways, but mitochondrial adaptation, regulation of energy homeostasis by AMPK, and lipid-mediated control of insulin signalling will remain part of future muscle research [19]. However, the complexity of muscles will require further multidimensional data integration and multidisciplinary cooperation. Finally, translation strategies for muscle-oriented research will need to reach out to society to deliver guideline-relevant results for implementation in population-based prevention and treatment of people at risk of, or with overt, diabetes.

Funding During the last 6 years, the work of MR has been supported by the Ministry of Science and Research of the State of North RhineWestphalia (MIWF NRW), the German Federal Ministry of Health (BMG), a grant of the Federal Ministry for Research (BMBF) to the German Center for Diabetes Research (DZD e.V.) and by grants from the European Community (EC), the European Foundation for the Study of Diabetes (EFSD), the Helmholtz Alliance to Universities (ICEMED), the German Research Foundation (DFG), the German Diabetes Association (DDG) and the Schmutzler-Stiftung.

Duality of interest MR declares that there is no duality of interest associated with this manuscript.

Contribution statement $\mathrm{MR}$ is responsible for the conception of this manuscript, drafting the article and approving the final version for publication.

\section{References}

1. Randle PJ (1966) Carbohydrate metabolism and lipid storage and breakdown in diabetes. Diabetologia 2:237-247

2. Szendroedi J, Phielix E, Roden M (2011) The role of mitochondria in insulin resistance and type 2 diabetes mellitus. Nat Rev Endocrinol 13:92-103

3. Samuel VT, Shulman GI (2012) Mechanisms for insulin resistance: common threads and missing links. Cell 148:852-871

4. Moltke I, Grarup N, Jørgensen ME et al (2014) A common Greenlandic TBC1D4 variant confers muscle insulin resistance and type 2 diabetes. Nature 512:190-193

5. Szendroedi J, Schmid AI, Meyerspeer M et al (2009) Impaired mitochondrial function and insulin resistance of skeletal muscle in mitochondrial diabetes. Diabetes Care 32:677-679

6. Szendroedi J, Yoshimura T, Phielix E et al (2014) Role of diacylglycerol activation of PKC $\theta$ in lipid-induced muscle insulin resistance in humans. Proc Natl Acad Sci U S A 111:9597-9602

7. Pillon NJ, Bilan PJ, Fink LN, Klip A (2013) Cross-talk between skeletal muscle and immune cells: muscle-derived mediators and metabolic implications. Am J Physiol Endocrinol Metab 304: E453-E465

8. Bork-Jensen J, Scheele C, Christophersen DV et al (2015) Glucose tolerance is associated with differential expression of microRNAs in skeletal muscle: results from studies of twins with and without type 2 diabetes. Diabetologia 58:363-373

9. Jin C, Henao-Mejia J, Flavell RA (2013) Innate immune receptors: key regulators of metabolic disease progression. Cell Metab 17: 873-882

10. Menche J, Sharma A, Kitsak M et al (2015) Disease networks. Uncovering disease-disease relationships through the incomplete interactome. Science 347:1257601

11. Kalyani RR, Corriere M, Ferrucci L (2014) Age-related and disease-related muscle loss: the effect of diabetes, obesity, and other diseases. Lancet Diabetes Endocrinol 2:819-829 
12. Cohen S, Nathan JA, Goldberg AL (2015) Muscle wasting in disease: molecular mechanisms and promising therapies. Nat Rev Drug Discov 14:58-74

13. Madden L, Juhas M, Kraus WE, Truskey GA, Bursac N (2015) Bioengineered human myobundles mimic clinical responses of skeletal muscle to drugs. Elife 4:e04885

14. Colberg SR, Sigal RJ, Fernhall B et al (2010) Exercise and type 2 diabetes: the American College of Sports Medicine and the American Diabetes Association: joint position statement executive summary. Diabetes Care 33:2692-2696

15. Hawley JA, Hargreaves M, Joyner MJ, Zierath JR (2014) Integrative biology of exercise. Cell 159:738-749
16. Kacerovsky-Bielesz G, Kacerovsky M, Chmelik M et al (2012) A single nucleotide polymorphism associates with responses of ATP synthesis to long-term exercise training in relatives of type 2 diabetic humans. Diabetes Care 35:350-357

17. Cooper AR, Sebire S, Montgomery AA et al (2012) Sedentary time, breaks in sedentary time and metabolic variables in people with newly diagnosed type 2 diabetes. Diabetologia 55:589-599

18. Carey AL, Kingwell BA (2009) Novel pharmacological approaches to combat obesity and insulin resistance: targeting skeletal muscle with 'exercise mimetics'. Diabetologia 52:2015-2026

19. Hue L, Taegtmeyer H (2009) The Randle cycle revisited: a new head for an old hat. Am J Physiol Endocrinol Metab 297:E578-E591 